\title{
Enhanced Visualization of the Fine Structure of the Stigmatic Surface of Citrus using Pre-fixation Washes
}

\author{
Hazel Y. Wetzstein ${ }^{1}$ \\ Department of Horticulture, University of Georgia, 1111 Miller Plant Science Building, Athens, \\ GA 30602
}

\author{
S. Edward Law \\ Department of Biological and Agricultural Engineering, The University of Georgia, Athens, \\ GA 30602
}

\begin{abstract}
ADDITIONAL INDEX wORDS. wet stigma, exudate, stigmatic papillae, scanning electron microscopy
Abstract. Stigma characteristics and morphology can be useful in taxonomic and phylogenetic studies, indicate relationships in stigma function and receptivity, and be valuable in evaluating pollen-stigma interactions. Problematic is that in some taxa, copious stigmatic exudate can obscure the fine structural details of the stigmatic surface. Such is the case for Citrus, which has a wet stigma type on which abundant exudate inundates surface papillae. The components of stigmatic surface compounds are highly heterogeneous and include carbohydrates, proteins, lipids, glycoproteins, and phenolic compounds. This study evaluated the efficacy of several pre-fixation wash treatments on removing surface exudate to visualize the underlying stigmatic surface. Wash treatments included various buffer solutions, surfactants, dilute acids/bases, and solvents. Stigmas prepared using conventional fixation methods in glutaraldehyde had considerable accumulations of reticulate surface deposits with stigmatic cells obscured. Pre-fixation washes containing solvents such as methanol, chloroform, and ethanol left accumulations of incompletely removed exudate and crystalline deposits. Alkaline water washes produced a crust-like deposit on stigma surfaces. Buffer washes left residues of plaquelike deposits with perforated areas. In contrast, excellent removal of stigmatic exudate was obtained with a pre-fixation wash composed of $0.2 \mathrm{M}$ Tris buffer, $\mathrm{pH}$ 7.2, containing $0.2 \%$ Triton X-100 surfactant and allowed clear imaging of the stigma and surface papillae morphology. A central sinus and radially arranged openings on the stigmatic surface were clearly visible and shown for the first time using scanning electron microscopy (SEM).
\end{abstract}

Prerequisites for successful sexual reproduction in higher plants include a sequence of critical interactions occurring between pollen and stigmatic surfaces. The function of the stigma is to capture pollen, facilitate pollen hydration, and support pollen germination and tube growth (Franklin-Tong, 2002; Herrero and Hormaza, 1996; Hiscock and Allen, 2008; Shivanna, 2003). The stigmatic surface varies widely in morphology and physiology. Classifications have been based on the extent of surface exudate and categorized into wet and dry types based on the presence or absence of fluid secretion on the stigmatic surface (Heslop-Harrison and Shivanna, 1977). The extent of secretions in stigmas can range from surface droplets to free-flowing liquid mantles engulfing stigmatic cells. In addition, stigmas can be classified by the location of the receptive surface, the degree of papillate cell development, and characteristics of the papillae such as cell number and degree of branching. Heslop-Harrison (1981) applied a classification based on morphological and physiological characters of the stigma to $\approx 1000$ species of some 900 genera of angiosperms. Certain large families exhibit stigma heterogeneity indicating that stigma characteristics may be useful in taxonomic and phylogenetic studies. Furthermore, stigma characteristics can indicate relationships in stigma function and receptivity, be valuable in evaluating pollenstigma interactions, and useful in predicting breeding systems (Carmo-Oliveira and de Morretes, 2009; Heslop-Harrison, 1981; Radcliffe et al., 2010; Shivanna, 2003). Assessments of

Received for publication 2 July 2012. Accepted for publication 25 July 2012. ${ }^{1}$ Corresponding author. E-mail: hywetz@uga.edu. structural changes in stigmatic cells also have been used to evaluate receptivity, flower longevity, and damage of agricultural chemicals in crop species (Wetzstein, 1990; Yi et al., $2003,2006)$. Problematic is that in some taxa, copious stigmatic exudate can obscure the fine details of the stigmatic surface in observations of both fresh and conventionally fixed preparations. Fixation, freeze-drying, and critical point drying can introduce severe artifacts.

Citrus has a wet stigma with copious exudate and a hollow style containing numerous canals (Ciampolini et al., 1981; Cresti et al., 1982; Distefano et al., 2011). Histochemical and biochemical analyses of the stigmatic exudate show that it is heterogeneous and composed of lipids, polysaccharides, and proteins. Cresti et al. (1982) evaluated the stigma of Citrus limon and using SEM evaluations of fresh tissues demonstrated that papillae were completely covered with surface exudate. Although extensive extraction of exudate compounds occurred after conventional fixation and critical point drying, papillae were still predominantly embedded in surface secretions with only apical portions of some stigmatic cells exposed.

Seedlessness is an important attribute in citrus that is desirable, especially in fruit used for fresh consumption, and is obtained in self-incompatible cultivars that exhibit parthenocarpic ability. However, seediness can arise when sexually compatible genotypes planted within proximity of parthenocarpic cultivars cross-pollinate flowers. The commercial appeal for citrus seedless fruit has resulted in a renewed interest in citrus reproductive biology including studies on self- and crossincompatibility of citrus accessions, pollen tube behavior, and 
pollination source effects on fruit quality and seediness (Chao, 2005; Distefano et al., 2009; Papadakis et al., 2009; Yamamoto et al., 2006). Strategies to prevent seed development have included agronomic spray applications that damage the stigmatic surface, inhibit pollen germination and tube growth, or impair fertilization (Mesejo et al., 2006, 2008). The presence of a copious exudate that obscures the stigmatic surface can limit evaluations of stigma fine structure, its relationship to pollen function, and stigma-pollen interactions.

Developing protocols to effectively remove stigmatic exudate in taxa in which structural details are obscured would be extremely useful in physiological, developmental, and taxonomic studies using microscopic analysis. With the goal of enhanced visualization of stigmatic surfaces, the objective of the current study was to evaluate the efficacy of different prefixation stigmatic wash treatments for removing surface exudate using citrus as a model. In addition, stigma washes applied before conventional fixation methods should maintain the structural integrity of stigmatic surface cells. The chemical diversity of components comprising stigmatic exudates indicates that substances may differ in polarity, molecular weight, and solubility. Wash treatments evaluated included various buffer solutions, surfactants, dilute acids and bases, and solvents. This article describes a method in which a pre-fixation wash composed of $0.2 \mathrm{M}$ Tris buffer, $\mathrm{pH} 7.2$, containing $0.2 \%$ Triton X-100 surfactant (Dow Chemical, Midland, MI) effectively removes stigmatic exudate and provides clear imaging of stigmatic surface papillae and morphology.

\section{Materials and Methods}

Flowers were obtained from mandarin orange trees (Citrus reticulata 'W. Murcott Afourer') maintained as potted plants. Flowers that had opened on the day of collection were selected; petals were unfurled and anthers had not yet dehisced (Fig. 1A).
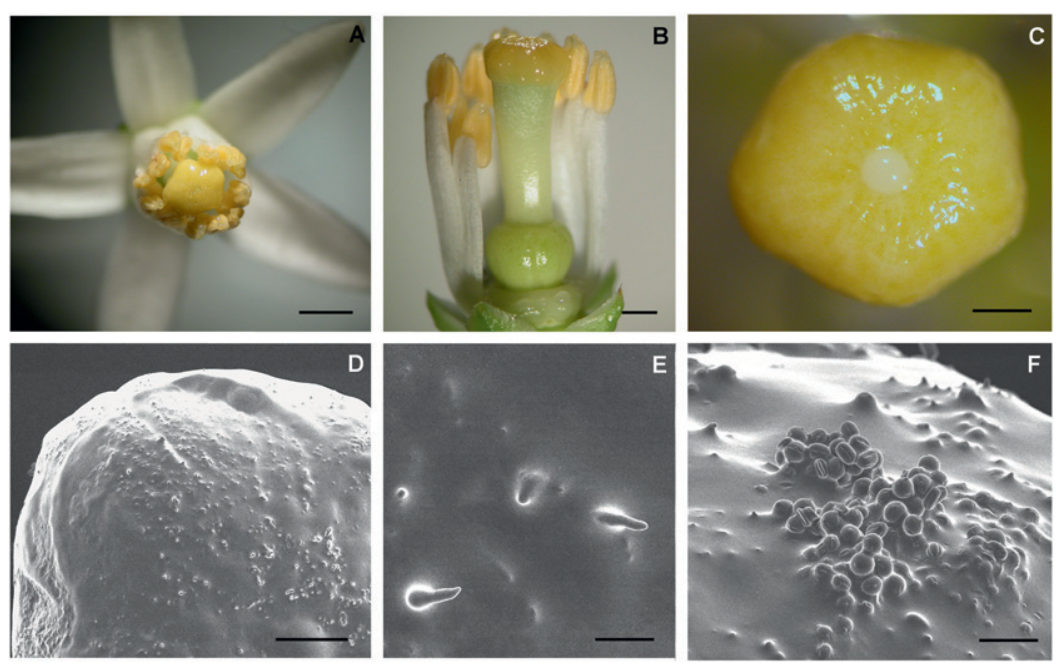

Fig. 1. Citrus reticulata flowers at anthesis. (A) Recently opened flower showing petals and stigma surrounded by anthers. (B) Lateral view of flower with petals and some stamens removed showing bulbous stigma, elongated cylindrical style, and ovary. (C) Stereomicroscope view of stigmatic surface covered with milky exudate that has accumulated in the central stigmatic region. (D) Scanning electron micrograph of a fresh unfixed stigma showing copious exudate on the surface. (E) Micrograph of the apical tips of a few papillae not completely covered by exudate. (F) Hand-pollinated stigma showing partial submersion of pollen and hydration of grains. Scale bars: $\mathbf{A}=2 \mathrm{~mm}, \mathbf{B}=1 \mathrm{~mm}, \mathbf{C}, \mathbf{D}=0.5 \mathrm{~mm}$, $\mathbf{E}=50 \mu \mathrm{m}, \mathbf{F}=100 \mu \mathrm{m}$.
Petals and stamens were removed, and the pistils were placed into $25 \mathrm{~mL}$ of one of the following wash solutions: 1) deionized water, $\mathrm{pH} 7.2 ; 2$ ) deionized water adjusted to $\mathrm{pH} 10.7$ with $1 \mathrm{~N}$ $\mathrm{NaOH}$; 3) $0.2 \mathrm{M}$ cacodylate buffer, $\mathrm{pH} 7.2$;4) $0.2 \mathrm{M}$ Tris buffer, $\mathrm{pH} 7.2 ; 5) 10 \%$ solution of ethanol:acetic acid $(3: 1 ; \mathrm{v} / \mathrm{v})$ fixative; 6) $50 \%$ methanol; 7) chloroform:methanol (1:2; v/v); 8) $0.2 \%$ Triton X-100 surfactant in water; 9) $0.2 \%$ Tween 20 (Vosco, Solon, OH) in water; 10) $0.2 \%$ Softcide Handsoap buffer, $\mathrm{pH} 7.2$ plus $0.2 \%$ Triton X-100. Stigmas were placed on an orbital shaker (Laboratory-Line Instruments, Melrose Park, IL) at $1500 \mathrm{rpm}$ for $16 \mathrm{~h}$. Preliminary studies indicated that ition and overnight washes were required to remove exudate. prepared using methods described by Wetzstein et al. (2011) for SEM. Tissues were fixed in $2 \%(\mathrm{v} / \mathrm{v})$ glutaraldehyde in $0.1 \mathrm{M}$ cacodylate buffer, $\mathrm{pH} 7.2$, washed in the same buffer, dehydrated dioxide using a critical point drier (Samdri-790; Tousimis Research, Rockville, MD). Dried samples were mounted on aluminum stubs using carbon conductive tabs and sputter-coated (SPI-Module; SPI Supplies, West Chester, PA) with gold. Japan) at $15 \mathrm{kV}$. Some tissues were fixed directly in glutaraldehyde with no washing pretreatments. In addition, fresh, nfixed, uncoated stigmas were immediately observed using the SEM at $10 \mathrm{kV}$.

\section{Results and Discussion}

The stigma in $C$. reticulata is a bulbous, yellow-colored structure at the apex of an elongated cylindrical style (Fig. 1B). The stigma is of the wet type (Heslop-Harrison, 1981), and at petal opening, the stigmatic surface is covered by copious milky exudate, which can pool at the central region (Fig. 1C). SEM evaluations of fresh, unfixed tissues show a rounded and generally smooth stigmatic surface containing only minor surface irregularities (Fig. 1D). Stigmas are covered with accumulated exudate. Minor projections on the surface visible at higher magnifications are the tips of stigmatic papillae in regions where stigmatic cells are incompletely submerged (Fig. 1E). Observations of pollinated stigmas show how the copious exudate serves to capture and hydrate pollen (Fig. 1F).

Corresponding SEM micrograph pairs at low and high magnification are shown of representative stigmas observed fresh or after conventional fixation and processing either with or without various pre-fixation washes (Figs. 2 and 3); similar results were observed in replicate stigmas within each treatment. Fresh stigmas observed directly without fixation or critical point drying (Fig. 2A-B) had copious exudate on the surface with pooled accumulations evident in the central region of the stigma. Papillae were for the most part embedded with only the tips of some cells exposed. Conventional SEM preparation methods, in which tissues were fixed directly in glutaraldehyde without pre-fixation 

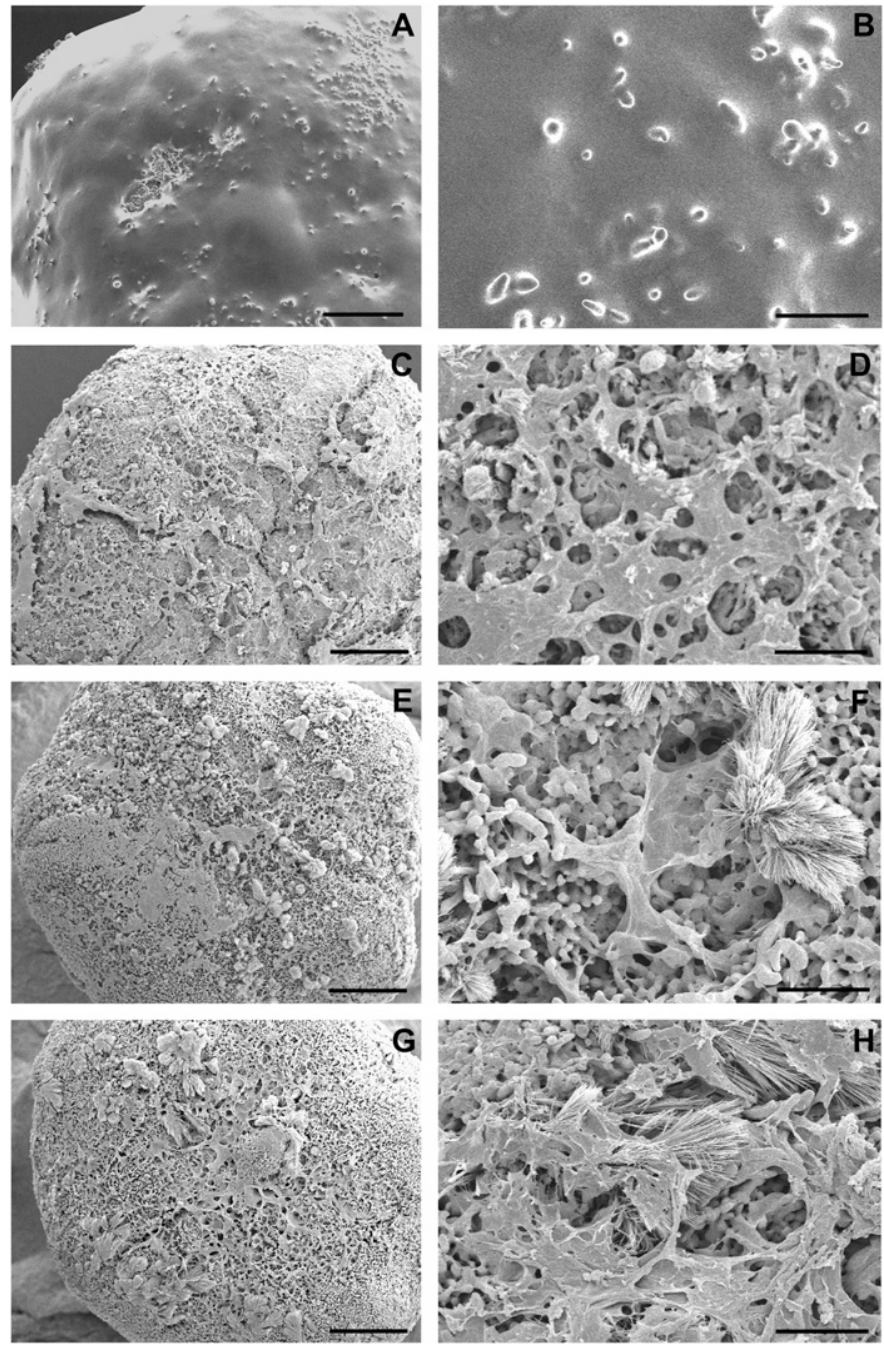

Fig. 2. Scanning electron micrograph of Citrus reticulata stigmas that were either observed fresh or fixed, dehydrated, and critical point dried after different prefixation treatments. (A) Surface view of a fresh unfixed stigma. (B) Higher magnification of a fresh, unfixed stigma showing copious exudate with occasional stigmatic papillae breaking the surface. (C) Stigma fixed directly in 2\% glutaraldehyde without washing. A central depression and radial indentions are visible; however, considerable amounts of exudate are retained on the surface. (D) Higher magnification of a sample prepared as in C. Papillae are obscured by perforated surface deposits and particulate material. (E) Stigma given a prefixation wash in $10 \%$ ethanol:acetic acid (3:1). The stigmatic surface has residual exudate. (F) Higher magnification of stigma shown in $\mathbf{E}$. Some stigma papillae are exposed, but veil-like exudate accumulations remain with crystalline deposits. (G) Stigma washed in 50\% methanol showing residual exudate. (H) Higher magnification of stigma shown in $\mathbf{G}$ with exudate and crystalline deposits. Scale bars: $\mathbf{A}, \mathbf{C}, \mathbf{E}, \mathbf{G}=0.5 \mathrm{~mm} ; \mathbf{B}, \mathbf{D}, \mathbf{F}, \mathbf{H}=100 \mu \mathrm{m}$.

washing, are shown in Figure 2C-D. Considerable amounts of exudate were extracted from stigmatic surfaces during processing. A central depression and radial indentions on the stigmatic surface were visible compared with fresh tissue observations (Fig. 2A) in which structures were obscured by a contiguous surface secretion. However, considerable amounts of exudate were retained on the surface, which included accumulations of reticulate deposits with perforated areas as well as granular and particulate materials (Fig. 2D). Stigmatic surface cells were not visible. The excellent fixation obtained when glutaraldehyde is used as a primary fixative in microscopy is attributed to the rapid crosslinking of the fixative with proteins, which causes stabilization
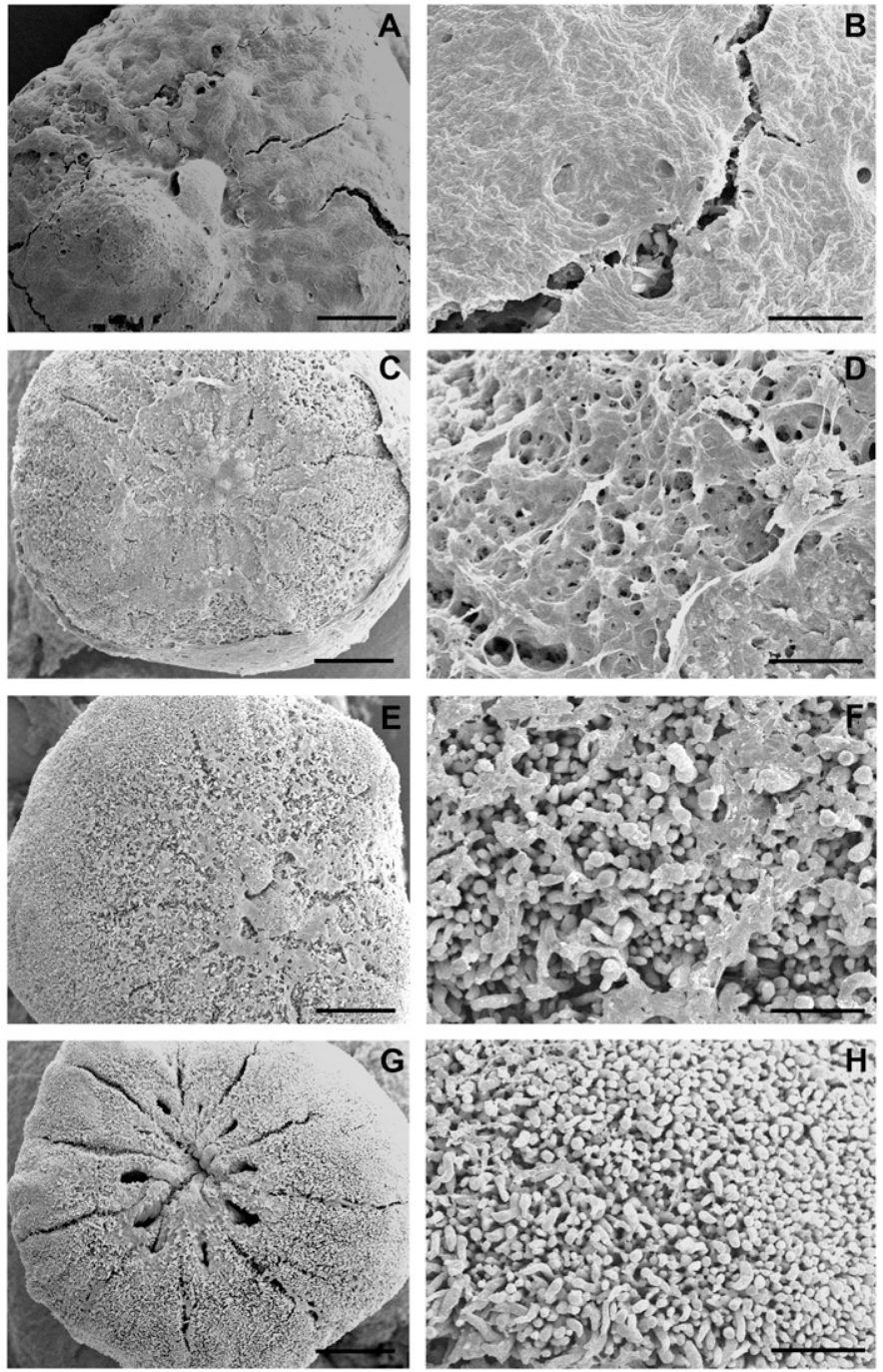

Fig. 3. Scanning electron micrograph of Citrus reticulata stigmas fixed, dehydrated, and critical point dried after different pre-fixation wash treatments. (A) Stigma given a pre-fixation wash in alkalinized water, $\mathrm{pH}$ 10.7, showing a central depression and a crust-like deposit on the surface. (B) Higher magnification of stigma shown in $\mathbf{A}$ at a region where a fissure has developed in the surface crust exposing papillae below. (C) Stigma washed in Tris buffer, $\mathrm{pH} 7.2$, showing partial removal of exudate. (D) Higher magnification of stigma shown in C. Papillae are still concealed by significant amounts of exudate residue. (E) Stigma given a pre-fixation wash in $0.2 \%$ Triton X-100. Some exudate has been removed; however, residual exudate remains. (F) Higher magnification of stigma shown in $\mathbf{E}$. Some stigma papillae are exposed, but some exudate accumulations remain. (G) Stigma washed with Tris buffer, $\mathrm{pH} 7.2$, containing $0.2 \%$ Triton X-100. A central sinus and radially arranged openings on the stigmatic surface are clearly visible. (H) Higher magnification of stigma shown in G. Stigma structure and papillae are clearly visible. Scale bars: A, C, E, $\mathbf{G}=0.5 \mathrm{~mm} ; \mathbf{B}, \mathbf{D}, \mathbf{F}, \mathbf{H}=100 \mu \mathrm{m}$.

of structures before extraction by buffers occurs (Glauert, 1975). The rationale for using the selected pre-fixation washes evaluated in the current study considered their potential extractability of stigmatic exudates in the absence of chemical binding.

Pre-fixation washes containing different solvents were evaluated for their potential in extracting lipophilic substances, which can be major constituents of stigma surface compounds. Washes containing solvents had limited effectiveness in extracting stigmatic surface exudates. Greater exposure of the stigmatic surface cells was obtained with pre-fixation washes of dilute ethanol-acetic acid (Fig. 2E-F) and methanol (Fig. 2G-H) 
when compared with tissues directly fixed with glutaraldehyde. Although some stigmatic papillae became visible, they were still obscured with veil-like accumulations remaining from incomplete exudate removal. Stigmatic surfaces developed numerous elongated, needle-like crystalline deposits in both diluted ethanolacetic acid and methanol pre-fixation washes that formed after washing and subsequent fixation in glutaraldehyde. Excessive browning and tissue injury were obtained using chloroform:methanol washes; thus, stigmas were not observed microscopically.

Stigmas given a pre-fixation wash in deionized water or alkalinized water, $\mathrm{pH}$ 10.7, developed a crust-like deposit that covered stigmatic surfaces. A thick shell-like coating was very marked with alkaline water washes; deposits conformed to the overall shape of the stigma with a central depression evident (Fig. 3A). Random, irregular fissures occurred at breaks in the crust; at regions where larger cracks developed, underlying papillae were visible (Fig. 3B). Somewhat greater exudate removal occurred with stigmas given neutral buffer washes (Tris or cacodylate buffers at $\mathrm{pH} 7.2$ ), but considerable amounts of residue remained on the surface. Partial removal of stigmatic exudate after a Tris buffer wash is shown (Fig. 3C-D). Papillae are still concealed by significant amounts of exudate residue. Surface secretions remained as plaque-like rugulose deposits with perforated areas. The pattern and extractability of surface exudate appears to be affected by $\mathrm{pH}$ as illustrated by the crustlike deposit that formed with alkalinized water washes compared with that observed with neutral buffer pre-fixation washes, which partially removed exudate but left considerable residue.

Washes containing different surfactants produced stigmas with improved removal of exudate compared with most other treatments. As shown in stigmas given a pre-fixation wash with $0.2 \%$ Triton X-100 (Figs. 3E-F), stigmatic papillae are clearly defined. Triton X-100 is a nonionic detergent containing both hydrophilic and hydrophobic groups. However, significant exudate accumulations remained that are clearly shown at higher magnification (Fig. 3F). Other surfactant treatments with handsoap and Tween 20 were similar (results not shown). In contrast, excellent removal of stigmatic exudate was obtained with a pre-fixation wash combining both Tris buffer and Triton X-100 (Fig. 3G-H). Triton $\mathrm{X}-100$ when used in combination with Tris buffer at $\mathrm{pH} 7.2$ readily extracted stigmatic exudate and was more effective than a Triton-X pre-fixation wash given alone. A central sinus and radially arranged openings on the stigmatic surface are clearly visible and show the continuity of the papillar surface with the stylar canals and central hollow channel corresponding to descriptions of sectioned mandarin citrus pistils (Distefano et al., 2011). These surface structures have not been previously shown using SEM because canal openings are otherwise filled with secretions in both fresh (Fig. 2A) and conventionally fixed (Fig. $2 \mathrm{C})$ stigmas. The fine structure of stigmas given a Tris buffer plus Triton X-100 wash had clearly defined, elongated, finger-like papillae; stigmatic surfaces were generally devoid of surface debris (Fig. 2H).

Copious stigmatic exudate can obscure the fine structural details of the stigmatic surface in both fresh and conventionally fixed SEM preparations. This report describes a treatment that can be used to enhance visualization of the fine structure of stigmatic surfaces masked by exudate. Exposure of citrus stigmas to a pre-fixation wash solution composed of $0.2 \mathrm{M}$ Tris buffer, $\mathrm{pH} 7.2$, containing $0.2 \%$ Triton X-100 effectively removed stigmatic exudate allowing clear imaging of stigma structure and surface papillae morphology. A central sinus and radially arranged openings on the stigmatic surface were clearly visible and shown for the first time using SEM.

\section{Literature Cited}

Carmo-Oliveira, R. and B.L. de Morretes. 2009. Stigmatic surface in the Vochysiaceae: Reproductive and taxonomic implications. Acta Botanica Brasilica 23:780-785.

Chao, C.-C.T. 2005. Pollination study of mandarins and the effect on seediness and fruit size: Implications for seedless mandarin production. HortScience 40:362-365.

Ciampolini, F., M. Cresti, G. Sarfatti, and A. Tiezzi. 1981. Ultrastructure of the stylar canal cells of Citrus limon (Rutaceae). Plant Syst. Evol. 138:263-274.

Cresti, M., F. Ciampolini, J.L. Went, and H.J. Wilms. 1982. Ultrastructure and histochemistry of Citrus limon (L.) stigma. Planta 156:1-9.

Distefano, G., A. Gentile, and M. Herrero. 2011. Pollen-pistil interactions and early fruiting in parthenocarpic citrus. Ann. Bot. (Lond.) 108:499-509.

Distefano, G., G. Las Casas, S. La Malfa, A. Gentile, E. Tribulato, and M. Herrero. 2009. Pollen behavior in different mandarin hybrids. J. Amer. Soc. Hort. Sci. 134:583-588.

Franklin-Tong, V.E. 2002. The difficult question of sex: The mating game. Curr. Opin. Plant Biol. 5:14-18.

Glauert, A.M. 1975. Practical methods in electron microscopy. NorthHolland, New York, NY.

Herrero, M. and J.I. Hormaza. 1996. Pistil strategies controlling pollen tube growth. Sex. Plant Reprod. 9:343-347.

Heslop-Harrison, Y. 1981. Stigma characteristics and angiosperm taxonomy. Nord. J. Bot. 1:401-420.

Heslop-Harrison, Y. and K.R. Shivanna. 1977. The receptive surface of the angiosperm stigma. Ann. Bot. (Lond.) 41:1233-1258.

Hiscock, S.J. and A.M. Allen. 2008. Diverse cell signaling pathways regulate pollen-stigma interactions: The search for consensus. New Phytol. 179:286-317.

Mesejo, C., A. Martinez-Fuentes, C. Reig, and M. Agusti. 2008. Gibberellic acid impairs fertilization in clementine mandarin under cross-pollination conditions. Plant Sci. 175:267-271.

Mesejo, C., A. Martinez-Fuentes, C. Reig, F. Rivas, and M. Agusti. 2006. The inhibitory effect of $\mathrm{CuSO}_{4}$ on Citrus pollen germination and pollen tube growth and its application for the production of seedless fruit. Plant Sci. 170:37-43.

Papadakis, I.E., E.E. Protopapadakis, and I.N. Therios. 2009. Yield and fruit quality of 'Nova' hybrid [Citrus clementina hort. ex. Tanaka $\times$ (C. reticulata Blanco $\times C$. paradisi Macfad)] and two clementine varieties (C. clementina hort. ex Tanaka) as affected by self- and cross-pollination. Sci. Hort. 121:38-41.

Radcliffe, C.A., J.M. Affolter, and H.Y. Wetzstein. 2010. Flower morphology and development in georgia plume, Elliottia racemosa (Ericaceae), a rare coastal plain endemic. J. Amer. Soc. Hort. Sci. 135:487-493.

Shivanna, K.R. 2003. Pollen biology and biotechnology. Science Publ., Enfield, NH.

Wetzstein, H.Y. 1990. Stigmatic surface degeneration and inhibition of pollen germination with selected pesticidal sprays during receptivity in pecan. J. Amer. Soc. Hort. Sci. 115:656-661.

Wetzstein, H.Y., N. Ravid, E. Wilkins, and A.P. Martinelli. 2011. A morphological and histological characterization of bisexual and male flower types in pomegranate. J. Amer. Soc. Hort. Sci. 136:83-92.

Yamamoto, M., T. Kubo, and S. Tominaga. 2006. Self- and crossincompatibility of various citrus accessions. J. Jpn. Soc. Hort. Sci. 75:372-378.

Yi, W., S.E. Law, D. McCoy, and H.Y. Wetzstein. 2006. Stigma development and receptivity in almond (Prunus dulcis). Ann. Bot. (Lond.) 97:57-63.

Yi, W., S.E. Law, and H.Y. Wetzstein. 2003. An in vitro study of fungicide effects on pollen germination and tube growth in almond. HortScience 38:1086-1088. 